\title{
INVESTIGATIONS ON TOPS INTERFEROMETRY WITH TERRASAR-X
}

\author{
Pau Prats, Luca Marotti, Steffen Wollstadt, Rolf Scheiber \\ Microwaves and Radar Institute \\ German Aerospace Center
}

\begin{abstract}
This paper presents results on SAR interferometry with the so-called TOPS mode. The rationale to retrieve accurate interferometric products with such a mode is expounded, emphasizing the critical step of coregistering the pairs. Due to the particularities of the TOPS mode, a high Doppler-centroid is present at burst edges, demanding very high azimuth coregistration performance. A coregistration accuracy of one tenth of a pixel, as it is usually recommended with interferometric applications, will result in a large undesired azimuth phase ramp in the TOPS mode, above all at X-band. This paper presents two approaches based on the spectral diversity technique to estimate this offset with the required accuracy. Experimental results with repeat-pass TerraSAR-X data are shown to validate the proposed approach.
\end{abstract}

Index Terms - TOPS, Interferometry, TerraSAR-X

\section{INTRODUCTION}

TOPS (Terrain Observation by Progressive Scans) has been proposed as a new wide-swath imaging mode [1]. It overcomes the problems of scalloping and azimuth-varying signal-to-ambiguity ratio of the conventional ScanSAR mode by means of steering the antenna in the along-track direction. To achieve the same swath coverage and avoid the undesired effects of ScanSAR, the antenna is rotated throughout the acquisition from backward to forward at a constant rotation rate, opposite to the spotlight case (see Fig. 1). The fast steering leads to a reduction of the observation time, and consequently a worsening of the azimuth resolution. However, now all targets are observed by the complete azimuth antenna pattern, and therefore the scalloping effect diminishes and the azimuth ambiguities and the signal-to-noise ratio become constant in azimuth. At the end of the burst, the antenna look angle is changed to illuminate a second subswath, pointing again backward. When the last subswath is imaged, the antenna points back to the first subswath, so that no gaps are left between bursts of the same subswath.

Fig. 2 shows the time-frequency diagram (TFD) of one TOPS burst. The total azimuth bandwidth spans several PRF

Work partially funded under ESTEC contract no. 22243/09/NL/JA.

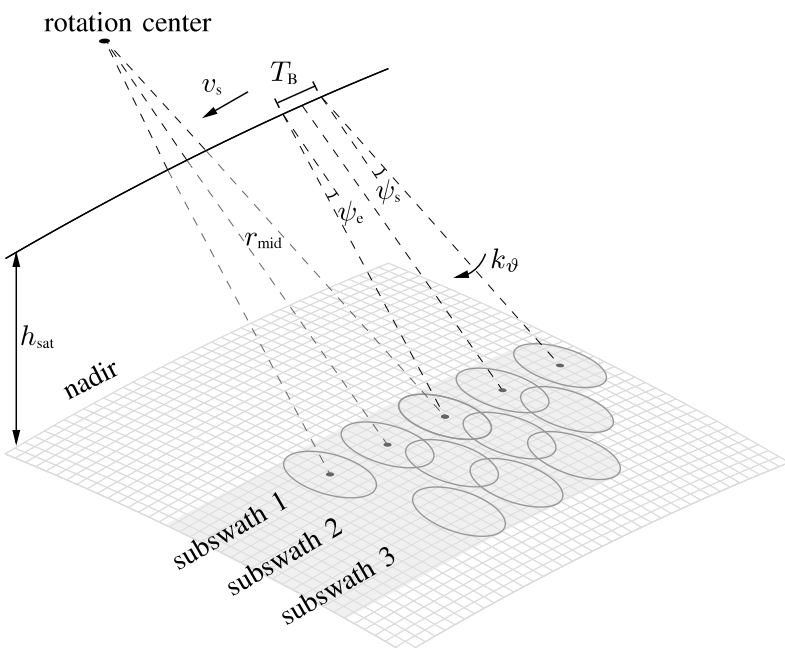

Fig. 1. Sketch of the TOPS acquisition geometry.

intervals, as in the spotlight case. Note also, that the rotation center is located behind the sensor, and as it happens in the ScanSAR mode, the focused burst is much larger than the raw data burst, requiring special care when performing the azimuth focusing. Note in Fig. 2 the dependence of the Doppler centroid on the azimuth position of the target within a burst, whose variation can reach several PRF intervals (the PRF is the gray area for a given time instant). This large Doppler variation will introduce severe requirements in the coregistration accuracy.

\section{TOPS INTERFEROMETRY}

\subsection{Coregistration Accuracy Requirements}

Similar as with ScanSAR, a precise knowledge of both the pointing accuracy and the along-track position are necessary in order to retrieve an interferometric pair with overlapping spectra. In a previous contribution [2] it was shown that one of the most challenging aspects in TOPS interferometry is the fact that the acquired data have large Doppler-centroid variations within a burst. It is well known that in presence of squint, linear phase ramps are induced in the focused response both in azimuth and range [3]. Thus, constant misregistration 


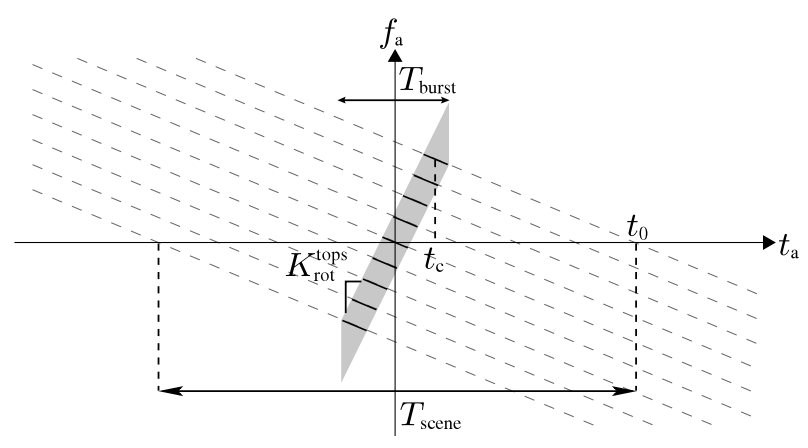

Fig. 2. Time frequency diagram in the TOPS mode.

can cause the presence of along-track and across-track linear phase ramps, of which the latter is in most cases negligible. However, since each TOPS burst is acquired with a varying Doppler centroid every focused point presents a different linear phase ramp in the azimuth direction

$$
\phi_{\mathrm{azerr}}=2 \pi f_{\mathrm{DC}} \Delta t
$$

where $f_{\mathrm{DC}}$ is the Doppler-centroid and $\Delta t$ is the coregistration error in seconds. Within a burst, this corresponds to a linear phase term along azimuth, since $f_{\mathrm{DC}}$ is a function of the azimuth position within the burst. An example of a typical TOPS TerraSAR-X acquisition can have a Doppler variation of about $5.4 \mathrm{kHz}$. With the given image sampling, a misregistration of 0.1 pixel spacing introduces a phase ramp of approximately $1.6 \pi$ within the burst. Therefore, an overall azimuth coregistration accuracy better than 0.001 of the pixel spacing is required for this configuration in order to achieve an error smaller than $3^{\circ}$.

\subsection{Fine Coregistration with Spectral Diversity}

Note that the above requirement applies mainly to a constant coregistration offset for the whole burst, and the achievable relative coregistration accuracy can be much better than this requirement. First, a coregistration can be performed either using orbit's information and an external DEM, amplitude cross-correlation, or coherence maximization, all of which yield accuracies better or around one tenth of a pixel. Indeed, a geometrical coregistration using an external DEM and the science orbits of TerraSAR-X yields a residual coregistration error in azimuth of only 0.005 samples in TOPS, which corresponds to the orbit accuracy of about $5 \mathrm{~cm}$ (and a sampling of $\approx 9 \mathrm{~m}$ ). Nevertheless, a fine coregistration is still necessary, which can be achieved using spectral diversity [4] with a large averaging window (all available data in a burst). Two possibilities are foreseen: the use of spectral diversity within a burst, or the use of spectral diversity in the overlapping region between two consecutive TOPS bursts. In the latter case, the spectral separation is much larger than the separation within the signal bandwidth due to the azimuth steering, and conse-
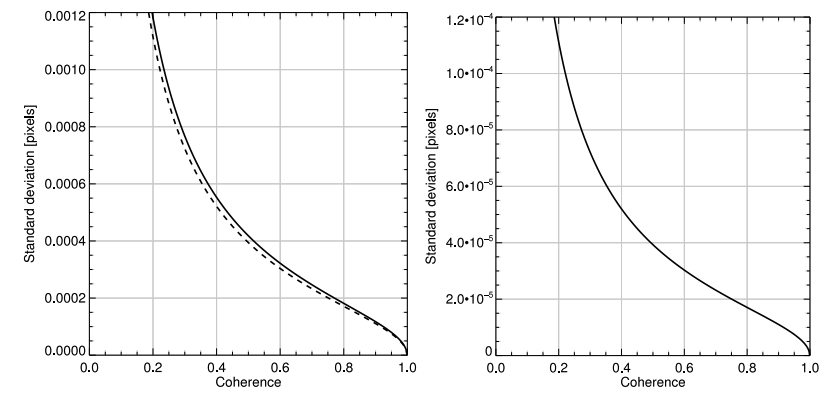

Fig. 3. (left) Error in the estimation of the constant azimuth coregistration error within one burst for different coherence values (the dashed line shows the Cramér-Rao bound [5]), and (right) the same error when using the overlap area between consecutive bursts. The values correspond to the Mexico City data take.

quently much higher accuracy can be obtained. The achievable accuracy in the estimation of the coregistration error with spectral diversity in image samples is given by [5]

$$
\sigma_{\mathrm{sd}}=\frac{\sqrt{2} \sigma_{\phi}}{2 \pi \Delta f} \frac{1}{\mathrm{dt}},
$$

where $\Delta f$ is the separation between the two spectral looks, $\mathrm{dt}$ is the image sampling in seconds, and $\sigma_{\phi}$ is the phase standard deviation of each spectral look given by [5]

$$
\sigma_{\phi}=\frac{1}{\sqrt{2 N / \alpha}} \sqrt{\frac{B}{b}} \frac{\sqrt{1-\gamma^{2}}}{\gamma}
$$

where $B$ is the processed bandwidth for a single target, $b$ is the look bandwidth, $N$ is the number of averaged samples, $\gamma$ is the interferometric coherence, and $\alpha$ is the oversampling factor. When using the first possibility, i.e. spectral diversity within one burst, it has been shown in [5] that when $b=B / 3$ $(\Delta f=B-b)$, eq. (2) approaches the Cramér-Rao bound in the estimation of the coregistration error. When using the second option, i.e. spectral diversity in the overlapping region between two consecutive bursts, the spectral separation $\Delta f$ in (2) is quite large and given by

$$
\Delta f_{\mathrm{ovl}}=\left|\frac{2 v_{\mathrm{eff}}^{2}}{\lambda \cdot\left(r_{\mathrm{rot}}-r\right)} T_{\text {cycle }}\right|,
$$

where $v_{\text {eff }}$ is the effective velocity, $r$ is the range distance, $r_{\text {rot }}$ is the rotation range, i.e. distance from the sensor to the rotation center, and $T_{\text {cycle }}$ is the cycle time of the TOPS acquisition.

Fig. 3 shows the expected performances for the two presented possibilities for a given TOPS data take. As expected, the accuracy obtained using the overlap area is better than using one burst, since the final accuracy improves inversely proportional to $\Delta f$, while only inversely proportional to the 
square root of the number of averaged samples. Nevertheless, the performance of both approaches is sufficient to estimate the desired value, even for very low coherence values. In any case, the amount of overlap also plays a role in the second approach and the final performance should be quantified for a given TOPS timeline. Besides the better performance of the second approach, it has also the advantage that the looks are already available, i.e. only the overlapping area of the left and right burst interferograms needs to be combined to generate the spectral diversity phase. This saves computational load when compared to the first approach, where the looks need to be generated explicitely. On the other hand, this approach has an important drawback: due to the larger sensitivity, the spectral diversity phase might be wrapped. The maximum coregistration error in pixels that can be measured without aliasing is $\Delta p_{\max }= \pm 1 /(2 \cdot \Delta f \cdot \mathrm{dt})$. For the particular case of the Mexico City results shown later, this value is equal to \pm 0.06 pixels. Therefore, it must be ensured that the residual coregistration error is smaller than this value, since otherwise a wrapped (wrong) value will be estimated. For TerraSAR-X this is not an issue, since a geometrical coregistration already leaves a smaller, as already commented.

\section{EXPERIMENTAL RESULTS}

Results acquired with TerraSAR-X over Mexico City (Sections 3.1 and 3.3) and the border Chile-Argentina border (Section 3.2) are shown next. In all cases, the TOPS acquisitions have been commanded with $16 \mathrm{~m}$ azimuth resolution and four sub-swaths (100 km ground-range). The number of bursts is 13 for the Mexico City data takes, and 14 for the ChileArgentina ones.

\subsection{Coregistration validation}

In order to validate the achievement of the coregistration requirements, the third sub-swath of the Mexico City data take has been selected. The interfeormetric pair has a time baseline of 22 days. Fig. 4 shows the coherence and the interferometric residual phases (after subtracting the SRTM DEM) before and after the refined coregistration procedure. A coregistration error of 0.05 samples was introduced to cleary observe the phase ramp, which corresponds to a $0.8 \pi$ phase variation along each burst. After the refined coregistration, the phase ramps have vanished. The remaining phase variations that can be observed are due to atmosphere, residual DEM errors, and deformation (see Section 3.3).

\subsection{InSAR Results}

Two TOPS repeat-pass data takes over the Chile-Argentina border have been used to evaluate the interferometric performance (mean coherence around 0.83). Fig. 5 shows the generated digital elevation model (DEM). A comparison with
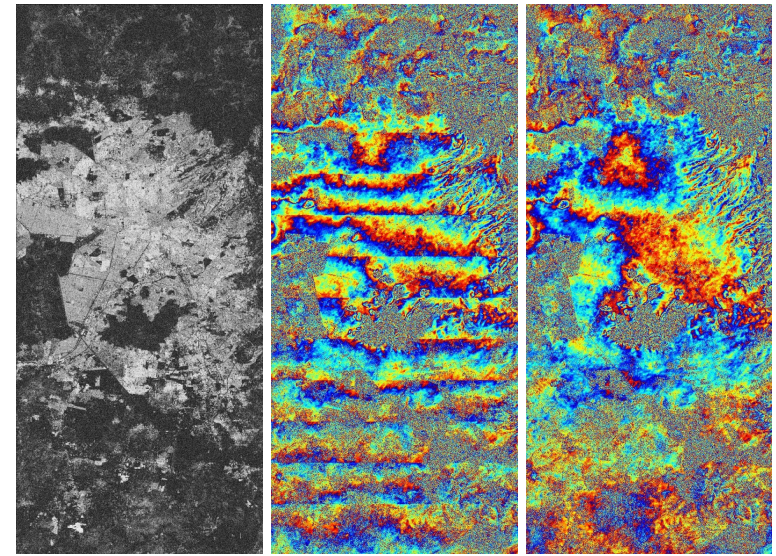

Fig. 4. TOPS interferometric coherence (left) and phases. (middle) With a coregistration error of 0.05 samples. (right) After using the first approach based on spectral diversity. The absolute azimuth coregistration error is better than 0.001 samples.

a stripmap DEM, also generated with repeat-pass TSX data, has been performed. The difference between both DEMs has a standard deviation of $15 \mathrm{~m}$, and no specific artefacts at burst edges can be observed. The perpendicular baselines are $118 \mathrm{~m}$ (TOPS) and $140 \mathrm{~m}$ (stripmap), yielding a height of ambiguity of $46 \mathrm{~m}$ and $39 \mathrm{~m}$, respectively. With these numbers, an atmospheric error of $5 \mathrm{~mm}$ would already produce $27 \mathrm{~m}$ in the DEM difference, which is considered to be the main source of error, as shown in Fig. 6.

\subsection{DInSAR Results}

A time series over Mexico City with the TOPS mode has been acquired by TerraSAR-X in a descending configuration interleaved with stripmap, which provides a repeat cycle of 22 days. Fig. 7 shows the subsidence measured in Mexico City when using a pair with 4 months difference. The image clearly shows the subsidence problem due to groundwater extraction [6]. Note that SRTM was used to remove the topography, while the atomspheric phase screen (APS) was not removed. Nevertheless, note that the maximum deformation is about one order of magnitude larger than that of the APS after 4 months.

\section{CONCLUSION}

This paper has presented special investigations concerning the coregistration requirements in the TOPS mode. Due to the high Doppler variation within a burst, a very precise azimuth coregistration is needed. Two options to achieve the requirements have been presented, namely the use of spectral diversity either within a burst or between consecutive bursts. In both cases, the required performance can be achieved, since 


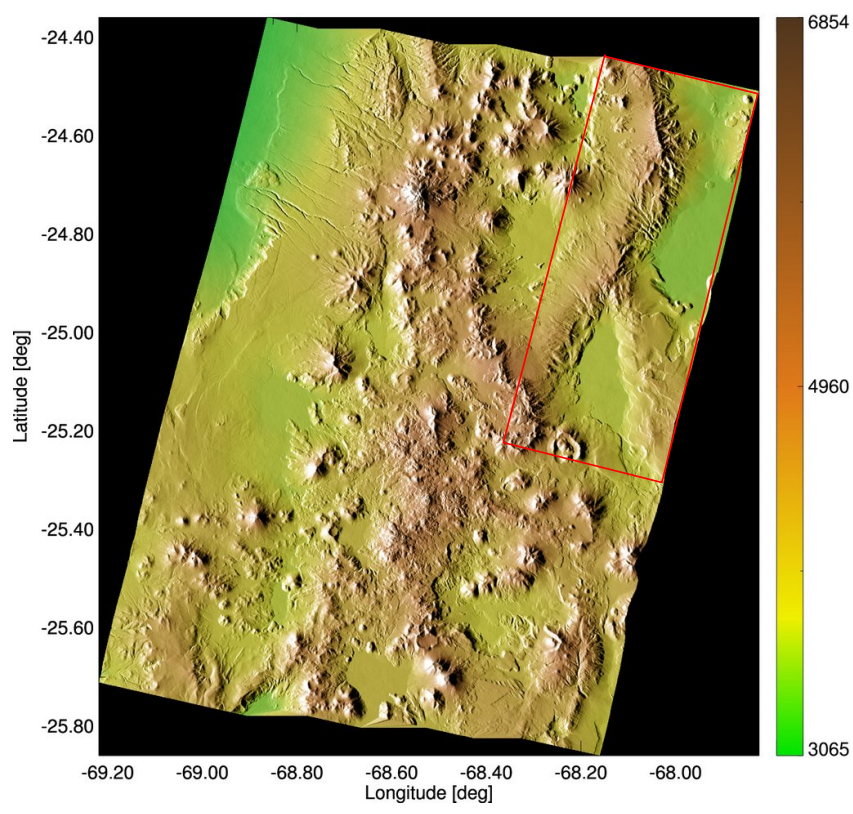

Fig. 5. DEM generated with two TOPS repeat-pass acqusitions. The red rectangle shows the area that has been used for comparison purposes with stripmap (see Fig. 6).

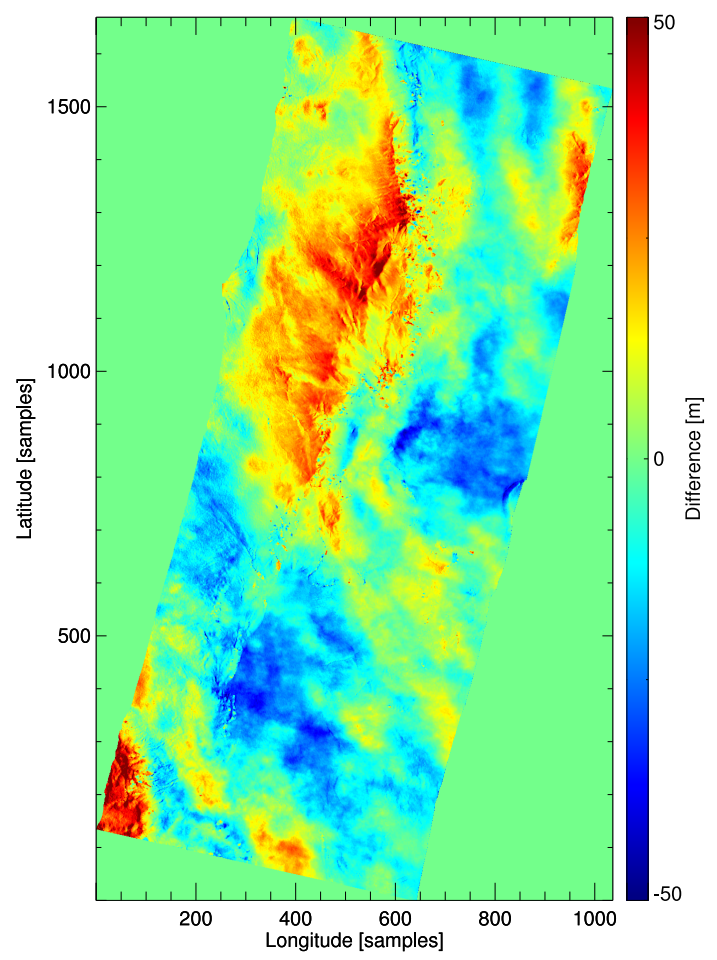

Fig. 6. Difference between TOPS and stripmap DEMs. Most of the errors seem to come from atmospheric artefacts.

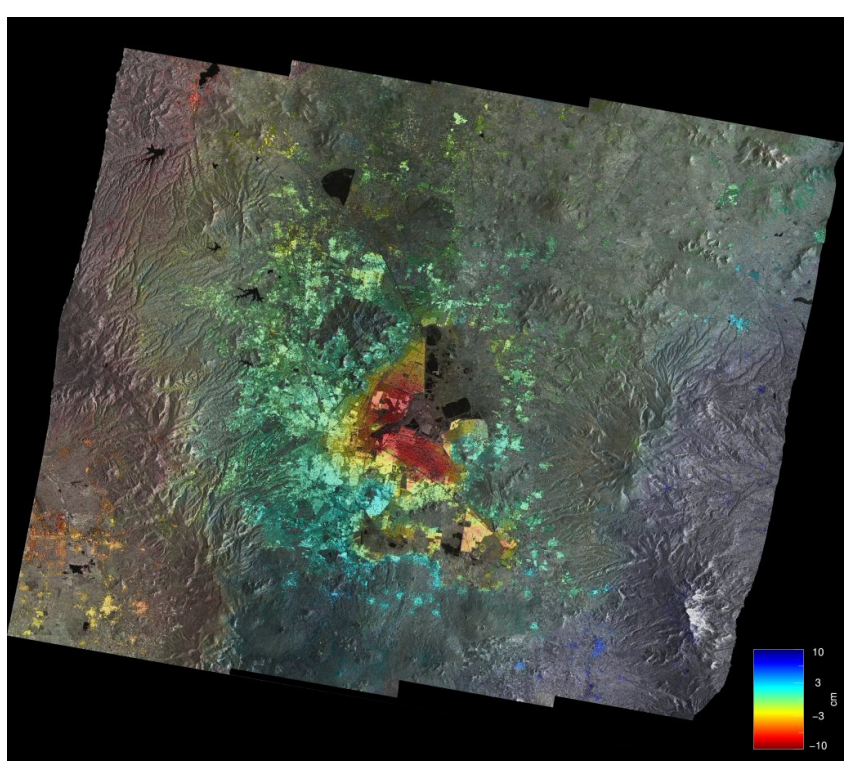

Fig. 7. Measured deformation over Mexico City with two TOPS TerraSAR-X images acquired with 4 months difference. A deformation greater than $10 \mathrm{~cm}$ can be observed in some city areas.

only a constant offset needs to be estimated after a previous coregistration step and, consequently, a large number of samples can be averaged. Indeed, a geometrical coregistration using an external DEM and the orbits is recommended, since thanks to the excellent knowledge of TerraSAR-X orbits, accuracies of about 0.005 pixels in azimuth in the TOPS mode can be reached. Results with TerraSAR-X data have been presented to validate the proposed approaches. Further work will include the study of point-like scatterers to measure the residual coregistration error.

\section{REFERENCES}

[1] F. De Zan and A. Monti Guarnieri, "TOPSAR: Terrain observation by progressive scans," IEEE Trans. Geosci. Remote Sensing, vol. 44, no. 9, pp. 2352-2360, Sept. 2006.

[2] P. Prats, L. Marotti, S. Wollstadt, and R. Scheiber, "TOPS interferometry with TerraSAR-X," in Proc. of European Conference on Synthetic Aperture Radar (EUSAR'10), Aachen, Germany, June 7-10 2010.

[3] M. Bara, R. Scheiber, A. Broquetas, and A. Moreira, "Interferometric SAR signal analysis in the presence of squint," IEEE Trans. Geosci. Remote Sensing, vol. 38, no. 5, pp. 2164-2178, Sept. 2000.

[4] R. Scheiber and A. Moreira, "Coregistration of interferometric SAR images using spectral diversity," IEEE Trans. Geosci. Remote Sensing, vol. 38, no. 5, pp. 2179-2191, July 2000.

[5] R. Bamler and M. Eineder, "Accuracy of differential shift estimation by correlation and split-bandwidth interferometry for wideband and Deltak SAR systems," IEEE Geosci. Remote Sensing Lett., vol. 2, no. 2, pp. 151-155, Apr. 2005.

[6] T. Strozzi, U. Wegmüller, C. L. Werner, A. Wiesmann, and V. Spreckels, "JERS SAR interferometry for land subsidence monitoring," IEEE Trans. Geosci. Remote Sensing, vol. 41, no. 7, pp. 1702-1708, July 2003. 